\title{
A novel artificial bee colony algorithm with an overall-degradation strategy and its performance on the benchmark functions of CEC 2014 special session
}

\author{
Bai Li ${ }^{1,2,3}$ \\ ${ }^{1}$ School of Control Science and Engineering, Zhejiang University, Hangzhou, 310027, China \\ ${ }^{2}$ School of Advanced Engineering, Beijing University of Aeronautics and Astronautics, Beijing, 100191, China \\ ${ }^{3}$ Department of Chemical Engineering, National Tsing Hua University, Hsinchu, 30013, Taiwan
}

Email address:

libai@zju.edu.cn

\section{To cite this article:}

Bai Li. A Novel Artificial Bee Colony Algorithm with an Overall-Degradation Strategy and Its Performance on the Benchmark Functions of CEC 2014 Special Session. Automation, Control and Intelligent Systems. Vol. 2, No. 5, 2014, pp. 71-80. doi: 10.11648/j.acis.20140205.11

\begin{abstract}
The artificial bee colony (ABC) algorithm has been a well-known swarm intelligence algorithm, which assimilates the cooperating behavior of bees when seeking for nectar sources. Aiming to improve the conventional $\mathrm{ABC}$ algorithm, we focus on the re-initialization phase. In this paper, an overall-degradation-oriented artificial bee colony (OD-ABC) algorithm is proposed, pursuing to fight against premature convergence. This is achieved through re-initializing majority of the employed bees at one time, rather than generating at most one scout bee in each iteration. In this work, our OD-ABC algorithm is compared against the conventional $\mathrm{ABC}$ algorithms using 24 benchmark functions that origin from the CEC 2014's competition on single objective real-parameter numerical optimization. The numerical results show that the OD-ABC algorithm is effective and thus can be employed to fight against premature convergence.
\end{abstract}

Keywords: Artificial Bee Colony, Numerical Optimization, CEC 2014 Competition, Overall Degradation Strategy, Evolutionary Algorithm

\section{Introduction}

Investigations on evolutionary algorithms have been the focus of research for decades [1], which are designed in general for the derivation of optimal or near-optimal solutions of the objective functions [2].

The Artificial bee colony $(\mathrm{ABC})$ is a swarm intelligence algorithm inspired by the foraging behavior of honey bees [3]. In this algorithm, the bee swarm mainly consists of three components, namely the employed bees, onlooker bees, and scout bees. In each cycle of iteration, the employed bees first carry out a global exploration. Those "qualified" employed bees then attract the onlooker bees to follow them. Due to the roulette selection strategy adopted in $\mathrm{ABC}$, the relative qualification of each employed bee is related to its corresponding probability of being followed by onlooker bees. At the end of each iteration, "unqualified" employed bee will perish and then a randomly re-initialized scout bee will take their places. It is worthwhile to notice that in the conventional $\mathrm{ABC}$ algorithm at most one scout bee can emerge in each iteration.

Several previous research studies on the basis of different numerical benchmark tests have confirmed that the $\mathrm{ABC}$ algorithm is competitive comparing to some other well-known evolutionary algorithms (e.g., genetic algorithm, differential evolution algorithm, ant colony optimization algorithm and particle swarm optimization algorithm) [4]. In addition, the framework of $\mathrm{ABC}$ is relatively simple and clear, making it easy to acquire satisfactory results at a low computational cost. Such merits have given rise to applications of $\mathrm{ABC}$ spanning across various areas, such as trajectory planning [5-7], structure optimization [8-10], clustering [11], machine learning [12], scheduling [13-16], image recognition [17-20] etc. 
Regarding the modifications ever made for the conventional $\mathrm{ABC}$, from the author's viewpoint, the prevailing ways can be broadly classified into three categories. Methods in the first category usually adopt some strategies or theories from the outside world (e.g., see Refs. [21] and [22]). Those so-called hybridized algorithms that combine $\mathrm{ABC}$ with exterior techniques fall in this category as well. The methods in the second category mainly focus on making some small changes on the search equations of the conventional ABC (e.g., see Refs. [23-25]). Regarding the modifications in the third category, some changes in the algorithm's framework should be carried out (e.g., see Refs. $[8,10,17,19,26,27])$. Here in this work, our interest is focused on improving the conventional $\mathrm{ABC}$ algorithm using a strategy that falls in the third category.

We notice that the conventional re-initialization process in $\mathrm{ABC}$ is not competent to handle premature convergence, especially when a "super individual" in a swarm emerges, which denotes a discovered local optimal location that attracting nearly all the bees to search around. In our enhanced re-initialization procedure, we do not restrict it to generate only one scout bee at each time. We intend to accumulate such inefficiency convergence information and make the required changes all at once. We call this an overall-degradation strategy. In this work, we proposed a hybrid ABC algorithm combined with such overall-degradation strategy (namely the OD-ABC algorithm).

The remainder of this paper is organized as follows. In Section 2, we review the fundamental principle of the conventional ABC algorithm. In Section 3, we present the motivation that has inspired us to improve the conventional $\mathrm{ABC}$ algorithm. Then in Section 4, we describe our OD-ABC algorithm, followed by Section 5 where the numerical tests as well as experimental results are presented. Thereafter, we discuss our findings in Section 6, and finally, the concluding remarks are provided in the last section.

\section{Conventional $A B C$ algorithm}

The conventional ABC algorithm employs three kinds of bees: scout bees searching for nectar sources randomly, employed bees associated with specific nectar sources, and onlooker bees following the guidance of employed bees. Typically, half of a bee colony would consist of the employed bees and the other half the onlooker bees [28].

At the very beginning, the scout bees are set out to randomly search for nectar sources. Thereafter, they are replaced by employed bees responsible for global exploration. During the global exploration procedure, those employed bees can share information (i.e., nectar source quality and the current location) with companions by means of "dancing". Then the onlooker bees select the nectar sources that the employed bees have discovered to exploit. It is worth pointing out that relatively higher-quality nectar sources are more likely to be chosen by the onlooker bees to exploit (as a natural consequence of utilizing the roulette selection strategy). If an employed bee finds no better nectar source than the one that it has previously discovered within a certain cycle, it turns into a scout bee again, which implies that its position will be randomly initialized in the search space.

A location of a nectar source represents a feasible solution to the problem, and the nectar quantity is reflected by the objective function value. Let $\mathrm{X}=\left(X^{1}, X^{2}, \cdots, X^{D}\right)_{1 \times D}$ represent a solution in the feasible solution space, $f u n(\cdot)$ be the objective function that needs to be minimized, $\operatorname{rand}(m, n)$ be a random number between $m$ and $n$ obeying the uniform distribution, and $S N$ be the population size of a bee swarm. As aforementioned, the number of onlooker bees in a bee colony is $S N / 2$, equalling that of the employed bees.

At first, as many as $S N / 2$ scout bees are randomly initialized in the feasible solution space. Equation (1) shows how the $j$ th element of the $i$ th scout bee's location $\mathrm{X}_{i}$ is calculated:

$$
\begin{aligned}
& X_{i}^{j} \leftarrow X_{\min }^{j}+\operatorname{rand}(0,1) \cdot\left(X_{\max }^{j}-X_{\min }^{j}\right), \\
& \qquad \quad i \in\{1,2, \ldots, S N / 2\}, j \in\{1,2, \ldots, D\},
\end{aligned}
$$

where $X_{\min }^{j}$ and $X_{\max }^{j}$ denote the lower and upper boundaries of this $j$ th element, and $D$ denotes the dimension of a feasible solution. Thereafter, the $S N / 2$ scout bees will become the employed bees and an iterated process begins from here.

In each cycle of iteration, an employed bee will share information with a randomly chosen companion and change one randomly chosen element of its location vector from $X_{i}^{j}$ to $X_{i}^{* j}$ using the following equation:

$$
\begin{aligned}
X_{i}^{* j} & \leftarrow X_{i}^{j}+\operatorname{rand}(-1,1) \cdot\left(X_{k}^{j}-X_{i}^{j}\right), \\
& k \in\{1,2, \ldots, S N / 2\}, j \in\{1,2, \ldots, D\}, k \neq i .
\end{aligned}
$$

It is necessary to note that $j$ and $k$ are both randomly selected integers. When all the employed bees arrive at their new nectar sources $\mathrm{X}_{i}^{*} i \in\{1,2, \ldots, S N / 2\}$, they evaluate the quality of these new nectars and then decide whether to stay at the new location or the previous one by means of a greedy selection strategy. Specifically, if the $i$ th employed bee finds that $f u n\left(\mathrm{X}_{i}^{*}\right)<\operatorname{fun}\left(\mathrm{X}_{i}\right)$, it will go to the new location $\mathrm{X}_{i}^{*}$, i.e., $\mathrm{X}_{i} \leftarrow \mathrm{X}_{i}^{*}$; otherwise, it remains at the previous location $\mathrm{X}_{i}$.

When all the employed bees have decided on their locations, a roulette selection strategy will direct the onlooker bees to select "qualified" employed bees to follow. A probability index $P$ is calculated according to Equations (3) and (4) to reflect the relative quality of nectar sources at which the employed bees are located. 


$$
\begin{gathered}
P(i)=\frac{\text { fitness }(i)}{\sum_{j=1}^{S N / 2} f i t n e s s(j)}, i \in\{1,2, \ldots, S N / 2\}, \\
\text { fitness }(i)=\left\{\begin{array}{ll}
\frac{1}{1+\operatorname{fun}\left(\mathrm{X}_{i}\right)} & \text { if } f u n\left(\mathrm{X}_{i}\right) \geq 0 \\
1+\left|\operatorname{fun}\left(\mathrm{X}_{i}\right)\right| & \text { if } \operatorname{fun}\left(\mathrm{X}_{i}\right)<0
\end{array} .\right.
\end{gathered}
$$

Each onlooker bee will search locally around an employed bee. For some $i$ th onlooker bee, a comparison is made between a random number $\operatorname{rand}(0,1)$ and $P(j)$. If $P(j) \geq \operatorname{rand}(0,1)$, this onlooker bee will search around the $j$ th employed bee; otherwise, a comparison between $\operatorname{rand}(0,1)$ and $P(j+1)$ will be made. If even $P(S N / 2)$ happened to be smaller than $\operatorname{rand}(0,1)$, such a comparison process is repeated from the first employed bee's $P(1)$ again until a larger $P(j)$ is found. Then, the corresponding $j$ th employed bee will be chosen. The following equation (i.e., Equation (5)) shows the location of the $i$ th onlooker bee $\mathrm{Y}_{i}=\left(X_{j}^{1}, \ldots, X_{j}^{k-1}, Y_{i}^{k}, X_{j}^{k+1}, \ldots, X_{j}^{D}\right)_{1 \times D}$ that searches locally around the selected $j$ th employed bee.

$$
\begin{aligned}
Y_{i}^{k} \leftarrow X_{j}^{k}+\operatorname{rand}(-1,1) \cdot\left(X_{m}^{k}-X_{j}^{k}\right), \\
\quad m \in\{1,2, \ldots, S N / 2\}, k \in\{1,2, \ldots, D\}, m \neq j .
\end{aligned}
$$

Note that in this equation $m$ and $k$ are randomly selected integers as well. When all the $S N / 2$ onlooker bees have determined their locations, a greedy selection strategy is implemented. This time, however, a comparison is made between $\operatorname{fun}\left(\mathrm{X}_{j}\right)$ and $\operatorname{fun}\left(\mathrm{Y}_{i}\right), i \in\{1,2, \ldots, S N / 2\}$. If $\operatorname{fun}\left(\mathrm{Y}_{i}\right)$ is smaller than $\operatorname{fun}\left(\mathrm{X}_{j}\right)$, the $j$ th employed bee will discard its current location $\mathrm{X}_{j}$ and fly to $\mathrm{Y}_{i}$, i.e., $\mathrm{X}_{j} \leftarrow \mathrm{Y}_{i}$; otherwise, the $j$ th employed bee remains at $\mathrm{X}_{j}$.

It is interesting to point out that every time the greedy selection is carried out, it involves one central employed bee. There is an index that is associated with each of the employed bees, namely trial, which memorizes inefficient search times that concerns each of the employed bees. Specifically, trial $(i)$ records the number of times that the $i$ th employed bee has searched inefficiently. That is, $\operatorname{trial}(i)$ is incremented by one each time when the condition $\operatorname{fun}\left(\mathrm{X}_{i}^{*}\right) \geq \operatorname{fun}\left(\mathrm{X}_{i}\right)$ or $\operatorname{fun}\left(\mathrm{Y}_{j}\right) \geq \operatorname{fun}\left(\mathrm{X}_{i}\right)$ is satisfied. At the beginning, each $\operatorname{trial}(i)$ is set to zero. As the iteration goes on, when trial $(i)$ reaches a predefined threshold Limit, the $i$ th employed bee will turn into a scout bee again.

\section{Motivation}

In this section, we elaborate on the reason why it is advisable to make some changes in the re-initialization phase of $\mathrm{ABC}$ [1].

At the end of each iteration, having just one scout bee at most to be generated during the re-initialization phase limits the capability of the algorithm to overcome premature convergence. As a matter of fact, it has been confirmed in some numerical experiments that directly discarding the scout bees will not necessarily deteriorate the convergence performance [29].

Particularly, when a "super individual" (i.e., a discovered local optimal location that attracting nearly all the bees to search around) emerges in a swarm, such re-initialization process is not efficient at all to overcome. Once an employed bee is re-initialized in one iteration, it is likely to be attracted back to the same local optimal location again since other companions are still gathering around that place.

The emergence of super individuals is one cause of premature convergence in swarm intelligence algorithms. Another chief cause may be that the search domain is too large, thus making the optimization process slow to converge. In such cases, re-initializating a majority (but not all) of bees in the colony will be a feasible way to improve the situation.

In the next section, we will introduce the OD-ABC algorithm in detail.

\section{Overall-degradation $A B C$ Algorithm}

The OD-ABC is different from the conventional ABC algorithm in the re-initialization phase. Here, before a new iteration begins (i.e., at the end of each iteration), any $\operatorname{trial}(i)$ that has exceeded Limit will be reset to Limit (rather than 0 ). Thereafter, average value of trial (i.e., $\frac{2}{S N} \sum_{i=1}^{S N / 2} \operatorname{trial}(i)$ ) is compared to $\alpha_{\text {odr }}$. Limit, where $\alpha_{\text {odr }} \in(0,1)$ is a user-specified scalar. If $\alpha_{\text {odr }}$. Limit is smaller than $\frac{2}{S N} \sum_{i=1}^{S N / 2} \operatorname{trial}(i)$, the whole swarm is considered to be not working efficiently to a degree of $\alpha_{o d r}$. Then, as many as round $\left(\alpha_{o d r} . S N / 2\right)$ randomly selected employed bees will be re-initialized according to Equation (1). At the same time, their corresponding trial indices should be reset to zero. If $\frac{2}{S N} \sum_{i=1}^{S N / 2} \operatorname{trial}(i)$ is smaller, the current iteration is terminated directly and a new iteration will begin.

The pseudo-code and a flowchart (see Fig. 1) of the OD-ABC algorithm are given as follows [1]. 


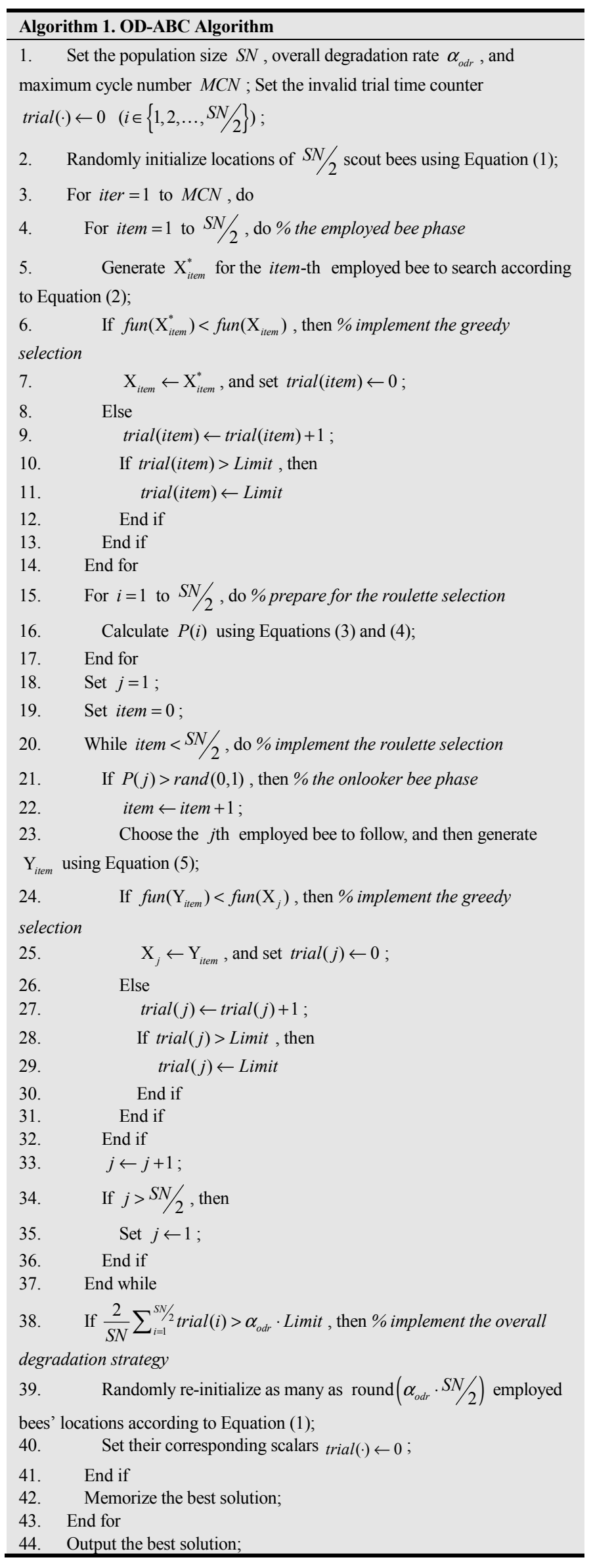

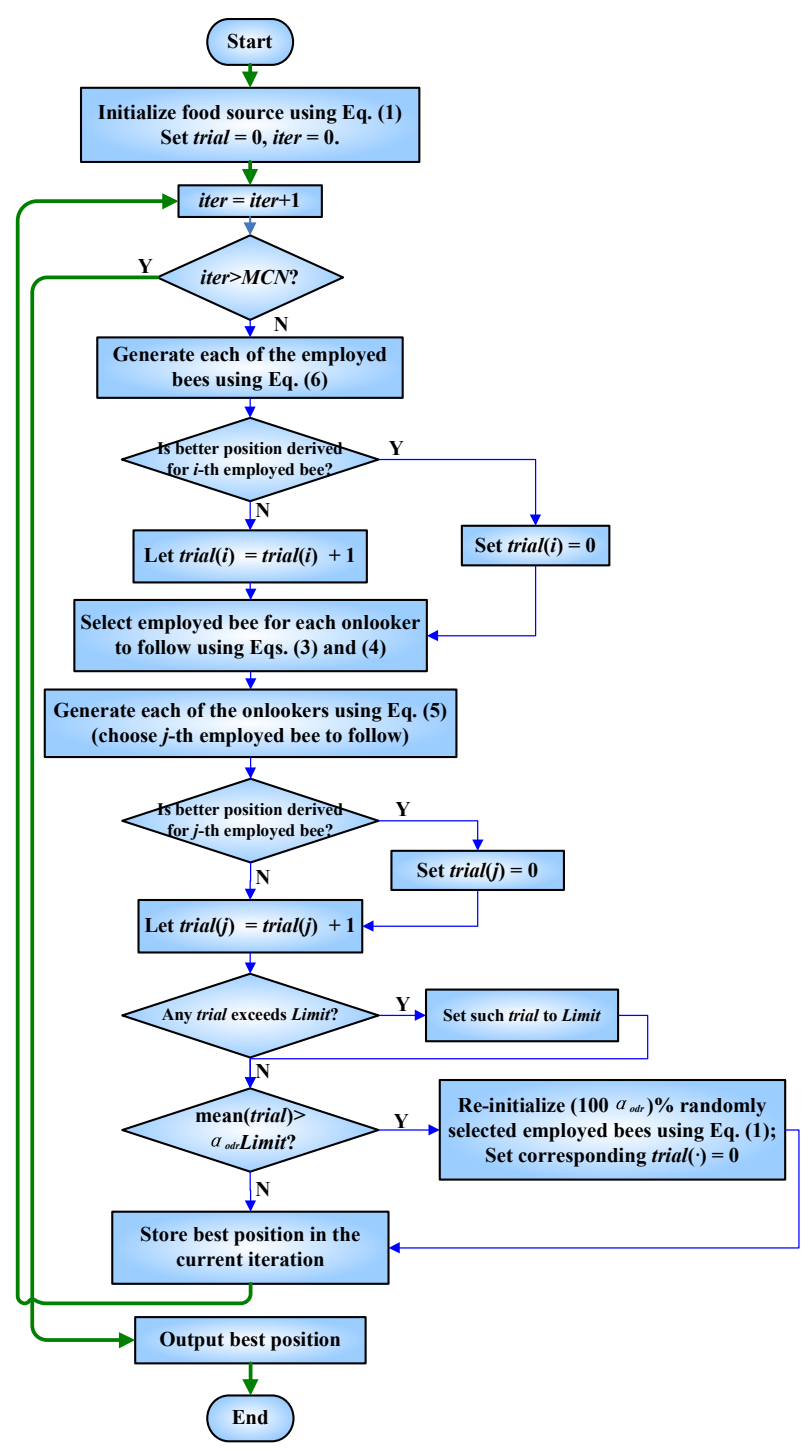

Fig 1. A flowchart of $O D-A B C$ algorithm.

\section{Experiments and Results}

In order to see the performance of the $\mathrm{OD}-\mathrm{ABC}$ algorithm in comparison with the conventional $\mathrm{ABC}$ algorithm, we systematically conducted a number of comprehensive simulation experiments (i.e., the first 24 benchmark functions for the competition of the CEC 2014 Special Session [30]). In all our computational experiments, the maximum number of cycles $M C N$ was constantly set to 5000 and the swarm population (i.e., $2 \cdot S N$ ) was set to 40 for both algorithms involved. Each of the experiments was repeated for 30 times with different random seeds. The search range is constantly set to $[-100,100]^{\text {Dim }}$, where $\operatorname{Dim}=50$ refers to the dimension of the benchmark problems in this work. All the simulations were carried out in a Matlab R2011b environment and executed on a Intel Core 2 Duo CPU with 2GB RAM running at $2.53 \mathrm{GHz}$ under the Microsoft Windows XP operating system.

The experimental results are listed in Table 1, where "Mean" denotes the average value at the 500th iteration from the 30 runs and S.D. is the corresponding standard deviation. 
In the last columns of this table, we report the statistical significance level of the difference of the means of the results produced by the best and the second best algorithms (with respect to their final accuracies). Note that here " $p$ value" reveals the chance if the null hypothesis (the differences between $\mathrm{OD}-\mathrm{ABC}$ and $\mathrm{ABC}$ can form a normal distribution) is true. '+' indicates the $\mathrm{OD}-\mathrm{ABC}$ works better than $\mathrm{ABC}$ at a
0.10 level of significance by two-tailed test; '-' indicates the $\mathrm{ABC}$ works better than $\mathrm{OD}-\mathrm{ABC}$ at a same level of significance; while ' ' indicates the two algorithm show no difference in statistics.

Three-dimensional visualization of a selected number of two-dimensional benchmark functions are illustrated in Figs. $2-10$.

Table 1. Result comparisons of $A B C$ and $O D-A B C$ on 24 benchmark functions in CEC 2014's competition.

\begin{tabular}{|c|c|c|c|c|c|c|}
\hline Test Function & $\begin{array}{l}\text { ABC } \\
\text { Mean } \\
\end{array}$ & S.D. & $\begin{array}{l}\text { OD-ABC } \\
\text { Mean } \\
\end{array}$ & S.D. & p-value & Significance \\
\hline$f 1$ & 6114570.5025 & 2419997.7581 & 6724954.4722 & 3359427.9062 & 0.3493 & . \\
\hline$f 3$ & 1818.6106 & 1719.7908 & 1355.3680 & 791.5116 & 0.1779 & . \\
\hline$f 4$ & 445.8312 & 29.4235 & 446.6215 & 31.2563 & 0.8774 & . \\
\hline$f 5$ & 520.1571 & 0.0236 & 520.0829 & 0.0186 & 0.0000 & + \\
\hline f6 & 614.8564 & 1.5735 & 615.1491 & 1.5120 & 0.5716 & . \\
\hline$f 7$ & 700.0009 & 0.0049 & 700.0006 & 0.0032 & 0.9426 & . \\
\hline$f 8$ & 800.0000 & 0.0000 & 800.0000 & 0.0000 & 1.0000 & . \\
\hline$f 9$ & 996.6754 & 14.1212 & 990.5091 & 14.9428 & 0.1846 & . \\
\hline$f 10$ & 1000.6780 & 0.5567 & 1000.7694 & 0.5598 & 0.6143 & . \\
\hline$f 11$ & 3255.2904 & 213.0970 & 3138.9450 & 311.3926 & 0.0656 & + \\
\hline$f 12$ & 1200.1958 & 0.0349 & 1200.1587 & 0.0245 & 0.0001 & + \\
\hline$f 14$ & 1400.1969 & 0.0227 & 1400.2157 & 0.0184 & 0.0011 & - \\
\hline$f 15$ & 1508.6140 & 1.5702 & 1508.9653 & 1.3481 & 0.5440 & . \\
\hline$f 16$ & 1609.9398 & 0.3453 & 1610.0154 & 0.3368 & 0.6143 & . \\
\hline$f 17$ & 2408523.2800 & 956030.9703 & 1841845.1394 & 1105817.4900 & 0.0300 & + \\
\hline$f 18$ & 2731.6489 & 749.3723 & 2480.4172 & 633.9575 & 0.3286 & . \\
\hline$f 19$ & 1907.3927 & 0.8670 & 1907.4452 & 0.7566 & 0.7655 & . \\
\hline$f 20$ & 11923.4498 & 5260.5395 & 17061.2679 & 7256.6336 & 0.0111 & - \\
\hline$f 21$ & 272118.8254 & 149638.8018 & 178289.7058 & 118298.2170 & 0.0098 & + \\
\hline$f 22$ & 2502.2540 & 129.5824 & 2570.8483 & 112.8751 & 0.0387 & - \\
\hline$f 23$ & 2615.3930 & 0.2305 & 2615.4770 & 0.4185 & 0.2134 & . \\
\hline$f 24$ & 2626.3521 & 6.6572 & 2628.2003 & 2.0630 & 0.0060 & - \\
\hline
\end{tabular}

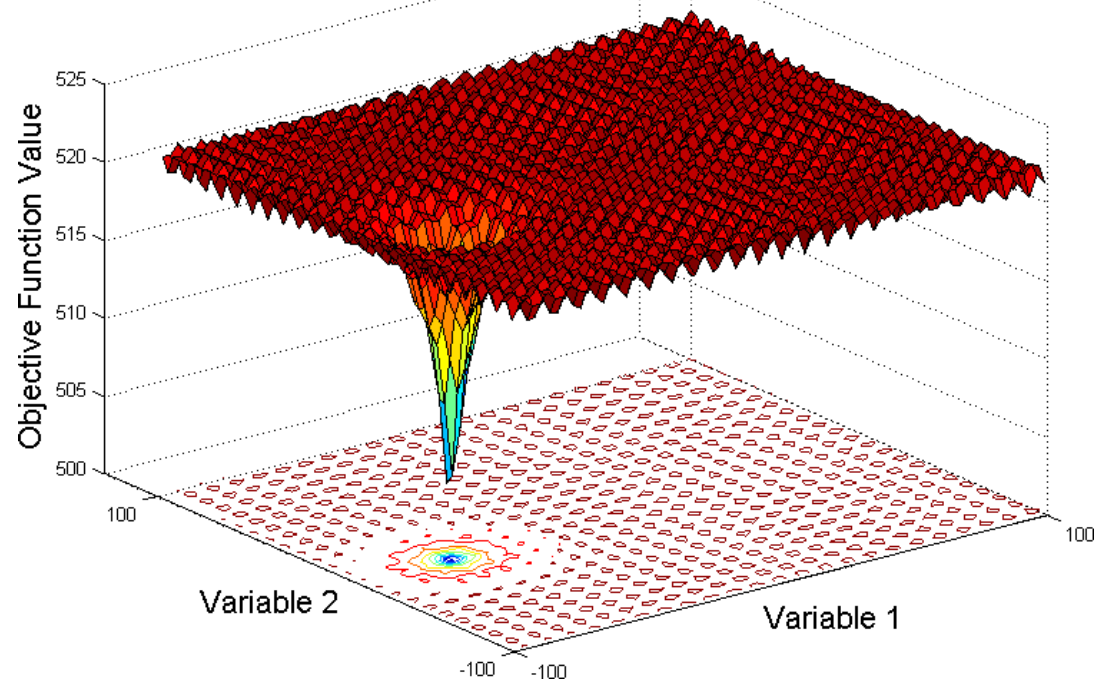

Fig 2. 3-D visualization for 2-D benchmark function 5. 


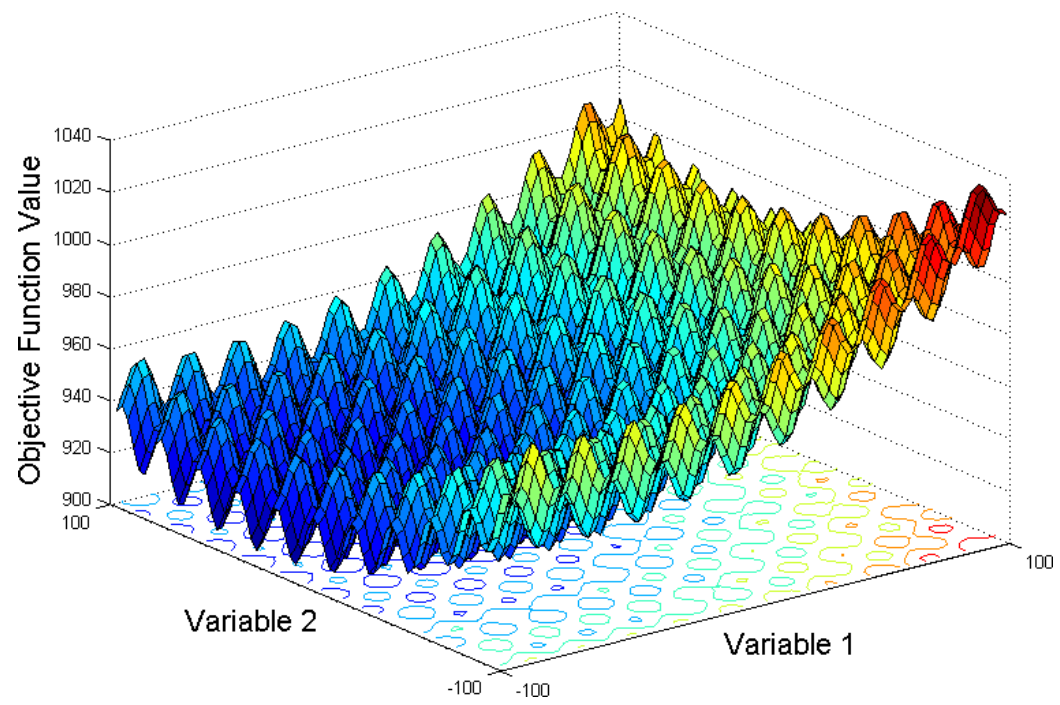

Fig 3. 3-D visualization for 2-D benchmark function 9.

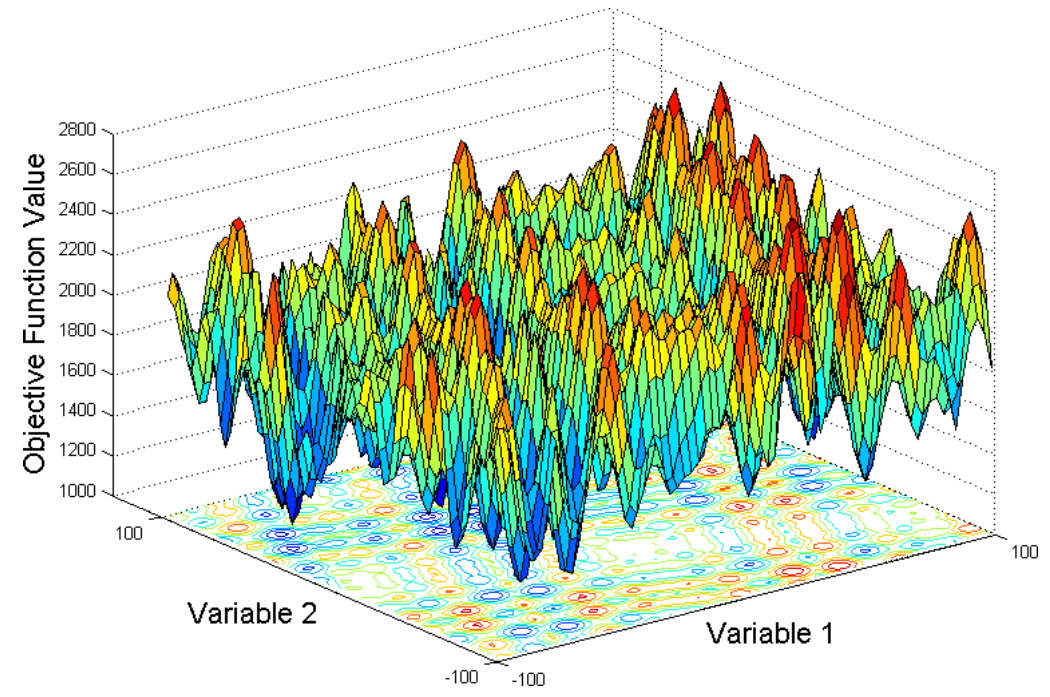

Fig 4. 3-D visualization for 2-D benchmark function 11.

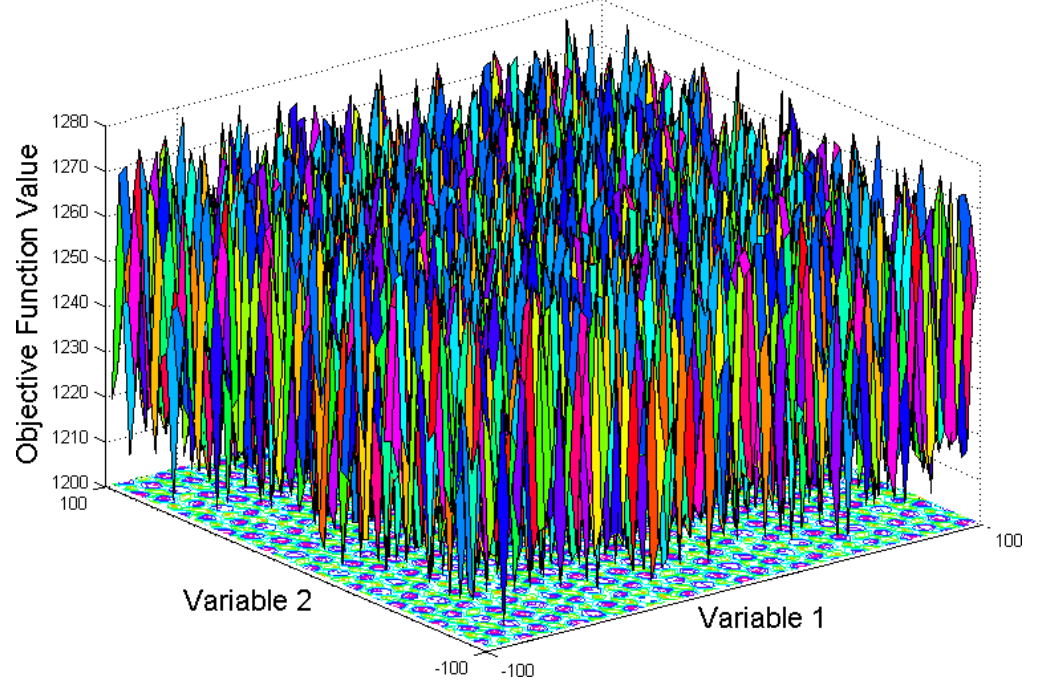

Fig 5. 3-D visualization for 2-D benchmark function 12 


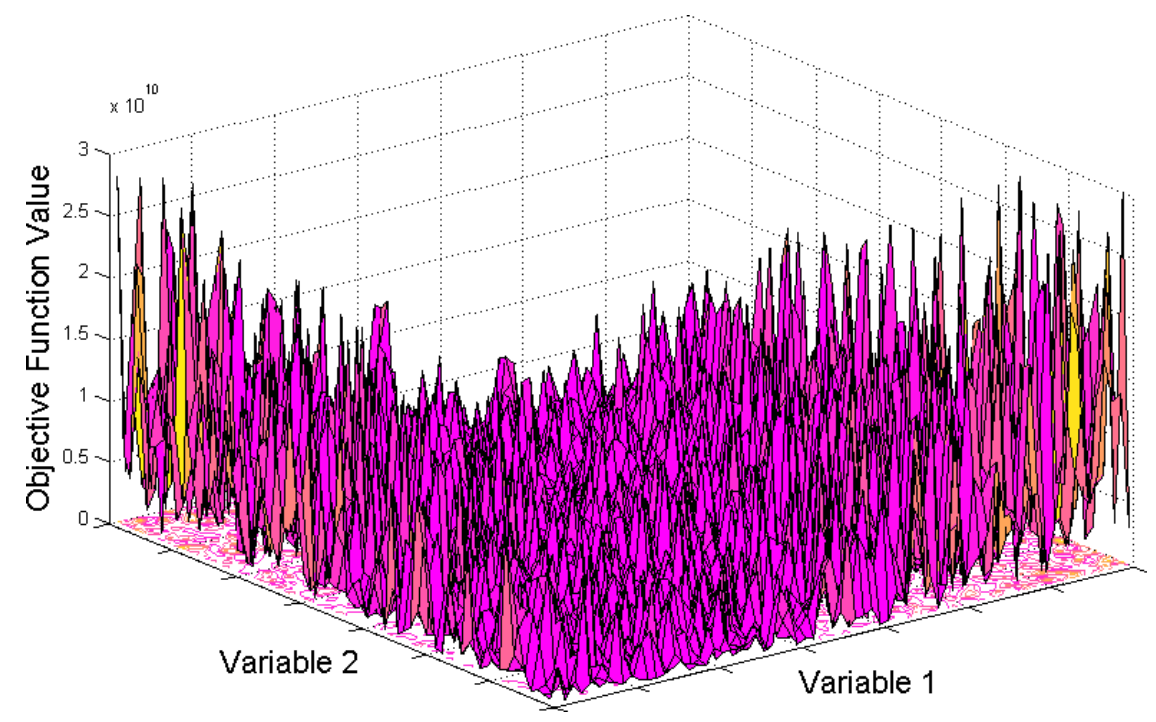

Fig 6. 3-D visualization for 2-D benchmark function 17.

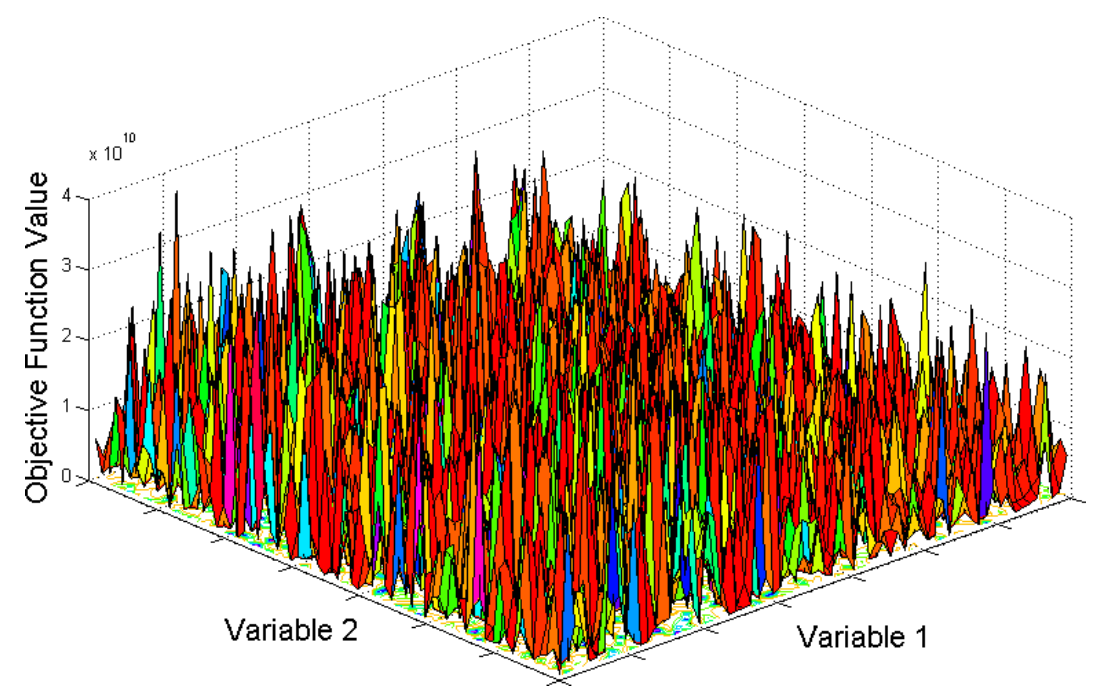

Fig 7. 3-D visualization for 2-D benchmark function 21 .

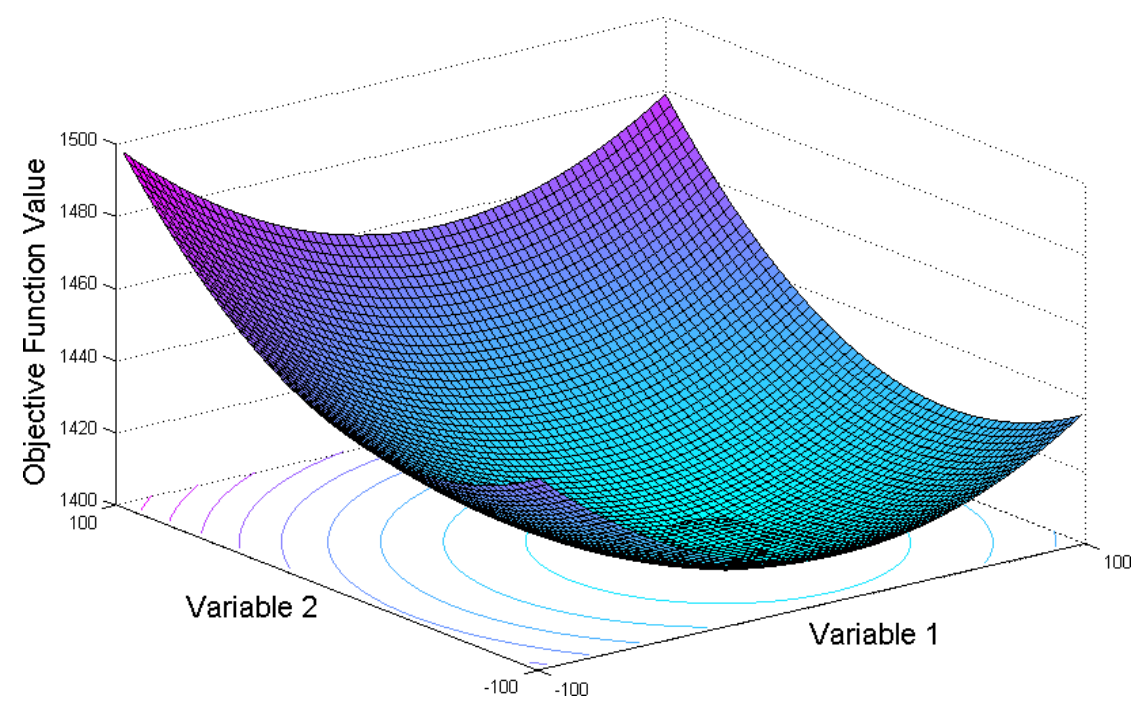

Fig 8. 3-D visualization for 2-D benchmark function 14. 


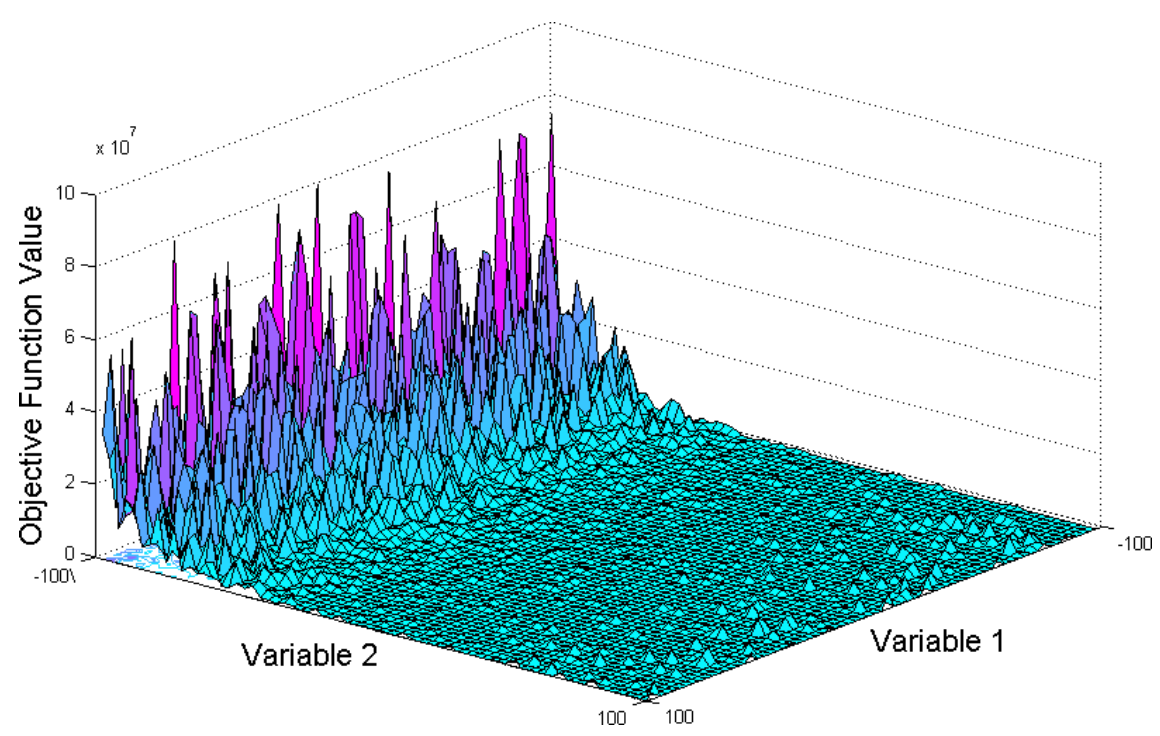

Fig 9. 3-D visualization for 2-D benchmark function 22 .

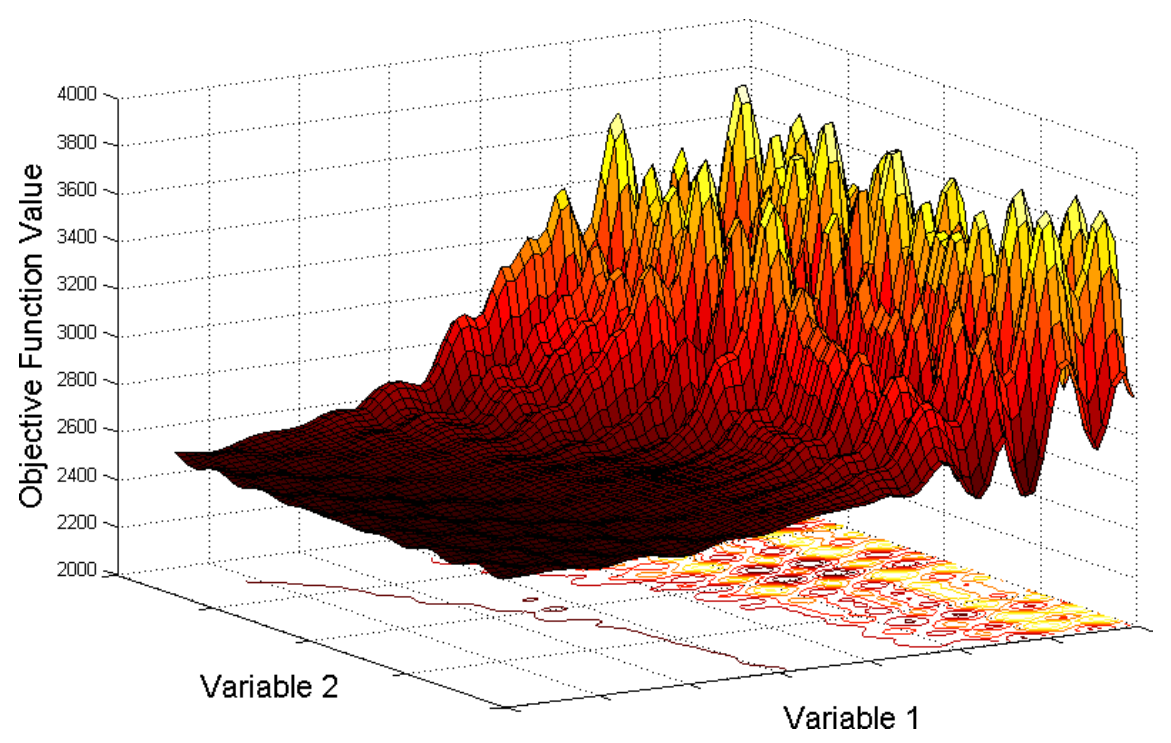

Fig 10. 3-D visualization for 2-D benchmark function 24 .

\section{Discussion}

According to the results listed in Table 1, we find that in most cases, the OD-ABC algorithm cannot outperform the conventional $\mathrm{ABC}$ algorithm with statistical significance. However, it is worthwhile to notice that the several benchmark functions that OD-ABC works better on (see Figs. 2-7) are far more complicated than the ones that $\mathrm{ABC}$ works better on (see Figs. 8-10). As in Figs. 2-7, there are in general a large number of local minimums around the global optimum, making it difficult to overcome premature convergence. In contrast, local minimums are not so close to the global optimums in the cases of benchmark functions 14, 22 and 24. All the mentioned above indicate that, the OD-ABC algorithm works well to handle the objective functions that are more challenging.

\section{Conclusion}

In this paper, we have proposed an OD-ABC algorithm, the main idea of which is to provide a more efficient re-initialization phase in the algorithm's framework. The innovations and highlights of in this work can be summarized as follows.

First, we have pointed out a critical issue that deserves well considered in the framework of the conventional ABC algorithm. Then, we provide a modification solution to this issue accordingly. Second, we adopt a state-of-the-art set of benchmark functions to test the performance of the concerns algorithms. The experimental results we obtained support our conclusion that the OD-ABC algorithm is efficient to fight against premature convergence. 
Despite these highlights and innovations, we confess there is still room for improvement since OD-ABC becomes inefficient when tested on some unimodal and/or simple multimodal benchmarks. As a feasible suggestion, combining such overall-degradation strategy with other existing strategies may work (e.g., see Refs. [1, 7, 31]).

After all, we believe that the unique and promising idea behind our OD-ABC algorithm is worth pursuing further.

\section{Acknowledgements}

The author declares that there is no conflict of interests regarding the publication of this paper. This work was supported in part by the 6th National College Students' Innovation \& Entrepreneurial Training Program in China under Grant No. 201210006050.

\section{References}

[1] B. Li, R. Chiong and R. Zhang, Balancing Exploration and Exploitation: An Analysis of the Balance-Evolution Artificial Bee Colony Algorithm, unpublished.

[2] D. Dasgupta and Z. Michalewicz (Eds.). Evolutionary algorithms in engineering applications. Springer Berlin Heidelberg, 1997.

[3] D. Karaboga and B. Akay, A modified artificial bee colony (ABC) algorithm for constrained optimization problems, Applied Soft Computing, Vol. 11, No. 3, pp. 3021-3031, 2011.

[4] D. Karaboga and B. Basturk, A powerful and efficient algorithm for numerical function optimization: artificial bee colony $(\mathrm{ABC})$ algorithm, Journal of global optimization, Vol. 39, No. 3, pp. 459-471, 2007.

[5] B. Li, L. G. Gong and C. H. Zhao, Unmanned combat aerial vehicles path planning using a novel probability density model based on Artificial Bee Colony algorithm, In 2013 Fourth International Conference on Intelligent Control and Information Processing (ICICIP 2013), pp. 620-625, IEEE, 2013.

[6] H. Duan, S. Shao, B. Su and L. Zhang, New development thoughts on the bio-inspired intelligence based control for unmanned combat aerial vehicle, Science China Technological Sciences, Vol. 53, No. 8, pp. 2025-2031, 2010.

[7] B. Li, L. G. Gong and W. L. Yang, An improved Artificial Bee Colony algorithm based on balance-evolution strategy for unmanned combat aerial vehicle path planning, The Scientific World Journal, Vol. 2014, No. 23704, pp. 1-10, 2014.

[8] B. Li, L. G. Gong and Y. Yao, On the performance of internal feedback artificial bee colony algorithm (IF-ABC) for protein secondary structure prediction. In 2013 Sixth International Conference on Advanced Computational Intelligence (ICACI 2013), pp. 33-38, IEEE, 2013.

[9] H. Sun, H. Luş and R. Betti, Identification of structural models using a modified Artificial Bee Colony algorithm, Computers \& Structures, Vol. 116, pp. 59-74, 2013.

[10] B. Li, Y. Li and L. G. Gong, Protein secondary structure optimization using an improved artificial bee colony algorithm based on AB off-lattice model, Engineering Applications of Artificial Intelligence, Vol. 27, pp. 70-79, 2014.
[11] R. J. Kuo, Y. D. Huang, C. C. Lin, Y. H. Wu and F. E. Zulvia, Automatic kernel clustering with bee colony optimization algorithm, Information Sciences, Vol. 283, pp. 107-122, 2014.

[12] B. Li, Research on WNN modeling for gold price forecasting based on improved Artificial Bee Colony algorithm, Computational intelligence and neuroscience, Vol. 2014, No. 270658, pp. 1-10, 2014.

[13] Q. K. Pan, M. Tasgetiren, P. N. Suganthan and T. J. Chua, A discrete artificial bee colony algorithm for the lot-streaming flow shop scheduling problem, Information sciences, Vol. 181, No. 12, pp. 2455-2468, 2011.

[14] J. Q. Li, Q. K.Pan and K. Z. Gao, Pareto-based discrete artificial bee colony algorithm for multi-objective flexible job shop scheduling problems, The International Journal of Advanced Manufacturing Technology, Vol. 55, pp. 1159-1169, 2011.

[15] L. Wang, G. Zhou, Y. Xu, S. Wang and M. Liu, An effective artificial bee colony algorithm for the flexible job-shop scheduling problem, The International Journal of Advanced Manufacturing Technology, Vol. 60, No. 4, pp. 303-315, 2012.

[16] M. F. Tasgetiren, Q. K. Pan, P. N. Suganthan and A. H. Chen, A discrete artificial bee colony algorithm for the total flowtime minimization in permutation flow shops, Information Sciences, Vol. 181, No. 16, pp. 3459-3475, 2011.

[17] B. Li, L. G. Gong and Y. Li, A Novel Artificial Bee Colony Algorithm Based on Internal-Feedback Strategy for Image Template Matching, The Scientific World Journal, Vol. 2014, No. 906861, pp. 1-14, 2014.

[18] C. Chidambaram and H. S. Lopes, An improved artificial bee colony algorithm for the object recognition problem in complex digital images using template matching, International Journal of Natural Computing Research, Vol. 1, No. 2, pp. 54-70, 2010.

[19] B. Li and Y. Yao, An edge-based optimization method for shape recognition using atomic potential function, Engineering Applications of Artificial Intelligence, Vol. 35, pp. 14-25, 2014.

[20] C. Xu and H. Duan, Artificial bee colony (ABC) optimized edge potential function (EPF) approach to target recognition for low-altitude aircraft, Pattern Recognition Letters, Vol. 31, No. 13, pp. 1759-1772, 2010.

[21] W. F. Gao, S. Y. Liu and L. L. Huang, A novel artificial bee colony algorithm with Powell's method, Applied Soft Computing, Vol. 13, No. 9, pp. 3763-3775, 2013.

[22] F. Kang, J. Li and Z. Ma, Rosenbrock artificial bee colony algorithm for accurate global optimization of numerical functions, Information Sciences, Vol. 181, No. 16, pp. 3508-3531, 2011.

[23] G. Zhu and S. Kwong, Gbest-guided artificial bee colony algorithm for numerical function optimization, Applied Mathematics and Computation, Vol. 217, No. 7, pp. 3166-3173, 2010.

[24] G. Q. Li, P. Niu and X. Xiao, Development and investigation of efficient artificial bee colony algorithm for numerical function optimization, Applied soft computing, Vol. 12. No. 1, pp. 320-332, 2012. 
[25] W. L. Xiang and M. Q. An, An efficient and robust artificial bee colony algorithm for numerical optimization, Computers \& Operations Research, Vol. 40, No. 5, pp. 1256-1265, 2013.

[26] A. Alizadegan, B. Asady and M. Ahmadpour, Two modified versions of artificial bee colony algorithm, Applied Mathematics and Computation, Vol. 225, pp. 601-609, 2013.

[27] B. Li and Y. Li, Y, BE-ABC: hybrid artificial bee colony algorithm with balancing evolution strategy, In 2012 Third International Conference on Intelligent Control and Information Processing (ICICIP 2012), pp. 217-222, IEEE, 2012.

[28] B. Li, R. Chiong and L. G. Gong, Search-Evasion Path Planning for Submarines Using the Artificial Bee Colony Algorithm, In Proceedings of the IEEE Congress on Evolutionary Computation (CEC 2014), pp. 528-625, IEEE, 2014.
[29] D. Karaboga and B. Basturk, B, On the performance of artificial bee colony (ABC) algorithm, Applied soft computing, Vol. 8, No. 1, pp. 687-697, 2008.

[30] J. J. Liang, B. Y. Qu and P. N. Suganthan, Problem definitions and evaluation criteria for the CEC 2014 special session and competition on single objective real-parameter numerical optimization, Technical Report 201311, Computational Intelligence Laboratory, Zhengzhou University, Zhengzhou China and Technical Report, Nanyang Technological University, Singapore, 2013..

[31] B. Li and Y. Li, A novel image matching method via lateral inhibition using balance-evolution artificial bee colony (BE-ABC) algorithm, submitted. 